\title{
Antifungal in vitro Activity of Essential Oils against Clinical Isolates of Malassezia pachydermatis from Canine Ears: A Report from a Practice Laboratory
}

\author{
Doris Bismarck $^{a} \quad$ Anika Dusold $^{a, b} \quad$ Anton Heusinger ${ }^{a} \quad E$ Elisabeth Müller \\ ${ }^{a}$ Laboklin GmbH \& Co. KG, Bad Kissingen, Germany; ${ }^{b}$ Coburg University of Applied Sciences and Arts, Coburg, \\ Germany
}

\section{Keywords}

Essential oils - Malassezia pachydermatis - Otitis externa · Veterinary medicine

\begin{abstract}
Background: The yeast Malassezia pachydermatis is a commensal but also the most isolated pathogenic yeast in canine otitis externa. Essential oils (EOs) exhibit antimicrobial activity and thus could be an alternative for treatment of otitis. Objective: The aim of this study was to analyse the in vitro efficacy of EOs against $M$. pachydermatis isolates from canine ears. Materials and Methods: Twenty-two EOs were tested for their in vitro activity against $15 \mathrm{M}$. pachydermatis isolates by agar disc diffusion. In addition, diluted EOs were tested and volatile antifungal activity was analysed by vapour assay. Results: Most EOs showed in vitro efficacy against $M$. pachydermatis. A different susceptibility pattern of tested isolates was found. Overall, the most potential EO was lemon grass. Also, cinnamon leaf, clove, manuka, Indian melissa, oregano, palmarosa, and winter savory oil exhibited a strong antifungal activity. Volatile activity was proven by the inhibiting effect in vapour assay. Conclusion: EOs show in vitro activity against $M$. pachydermatis. This study gives scientific background for the use of EOs in veterinary medicine. Diverse susceptibility patterns of different $M$. pachydermatis strains emphasise the helpfulness of an aromatogram as one lead for the best choice of an EO for therapy.
\end{abstract}

(C) 2019 The Author(s)

Published by S. Karger AG, Basel

karger@karger.com

www.karger.com/cmr

Karger $\stackrel{\text { '⿳亠丷厂 }}{=}$

Karger 2019 The Author(s)
Published by S. Karger AG, Basel $\quad$ Open acce

This article is licensed under the Creative Commons AttributionNonCommercial-NoDerivatives 40 International License (CC BYNC-ND) (http://wwwkarger.com/Services/OpenAccessLicense) Usage and distribution forger.com/Services/OpenAccessLicense). Usage and distribution for commercial purposes as well as any dict

\begin{abstract}
Antimykotische In-vitro-Wirksamkeit ätherischer Öle gegen Malassezia pachydermatis isoliert aus Hundeohren: Ein Bericht aus dem Routinelabor
\end{abstract}

\section{Schlüsselwörter}

Ätherische Öle · Malassezia pachydermatis · Otitis externa . Veterinärmedizin

\section{Zusammenfassung}

Einleitung: Der Hefepilz Malassezia pachydermatis gilt als Kommensale, ist aber gleichzeitig auch der am häufigsten isolierte Hefepilz bei caniner Otitis externa. Ätherische Öle könnten aufgrund ihrer antimikrobiellen Eigenschaften als alternative Therapie der Otitis verwendet werden. Zielsetzung: Ziel dieser Studie war die Untersuchung der antifungalen In-vitro-Wirksamkeit ätherischer Öle gegen M. pachydermatis-Isolate aus Hundeohren. Material und Methoden: Die In-vitro-Aktivität von 22 ätherischen Ölen wurde mittels Agar-Plättchen-Diffusionstest gegen 15 M. pachydermatis-Isolate untersucht. Zusätzlich wurden Verdünnungen der ätherischen Öle getestet und die volatile Wirkung mittels "vapour assay" analysiert. Ergebnisse: Die meisten ätherischen Öle zeigten in vitro eine Wirksamkeit gegen M. pachydermatis. Es konnte eine unterschiedliche Empfindlichkeit einzelner M. pachydermatis-Isolate gefunden werden. Lemongrasöl erwies sich als das po- 
tenteste ätherische Öl. Ebenfalls zeigten ZimtblätterNelken-, Manuka-, indisches Melissen-, Oregano-, Palmarosa- und Bergbohnenkrautöl eine starke antifungale Wirksamkeit. Die volatile Wirkung konnte mittels "vapour assay" gezeigt werden. Schlussfolgerung: In vitro zeigen ätherische Öle eine Wirksamkeit gegen den Hefepilz $M$. pachydermatis. Diese Studie bietet eine wissenschaftliche Basis zur Anwendung ätherischer Öle in der Veterinärmedizin. Die unterschiedliche Empfindlichkeit der verschiedenen $M$. pachydermatis-Isolate gegenüber einzelner ätherischer Öle zeigt einmal mehr die Nützlichkeit eines Aromatogramms als Anhaltspunkt bei der Auswahl des geeigneten ätherischen Öls für die Therapie.

(0)2019 The Author(s)

Published by S. Karger AG, Basel

\section{Introduction}

The yeast Malassezia pachydermatis is a commensal of the skin and mucosae of dogs. As an opportunistic secondary pathogen it is also the most significant yeast in canine otitis externa [1-3]. The mechanisms of the transition of $M$. pachydermatis from a commensal to a pathogen are not fully understood [4]. Generally, treatment of Malassezia-associated otitis externa is based on topical application of an antifungal drug combined with antibiotics to control bacterial infection and glucocorticoids to reduce inflammation $[4,5]$.

Standardised antifungal susceptibility testing of $M$. pachydermatis has not been established yet, but studies show that Malassezia isolated from animals with dermatitis lesions show less susceptibility to some fungal agents than isolates from healthy animals [6]. One possibly resistant isolate of $M$. pachydermatis to the antifungals ketoconazole and itraconazole was also described [7]. In vitro cross-resistance of Malassezia among azoles was observed [8]. In human medicine, general emergence of antifungal resistance is already known [9]. Besides, the interest in natural plant products in veterinary medicine is increasing [10-12]. Thus, essential oils (EOs) could be a candidate for alternative treatment of otitis externa. EOs are plant secondary metabolites which are secreted and stored in glandular trichomes, modified epidermal hairs of the plant, but also in pockets or reservoirs and even in intercellular spaces. EOs are known to protect the plant against enemies, such as pathogenic microorganisms, insects acting as plaque vectors, and herbivores. On the other hand, they can be beneficial for attracting insects necessary for dispersion of pollens and seeds [13, 14]. EOs are products made by water or steam distillation, mechanical processing, or dry distillation of plant material [15]. They are complex mixtures of volatile lipophilic natural compounds with a specific odour. They mainly consist of terpenes and/or phenylpropanoids [16]. Al- ready in ancient civilisations like Egypt, India, and China EOs were used for healing. Modern medical aromatherapy was founded by René-Maurice Gatefossé, who started utilising EOs in medical treatment in modern times. Inhalation, local treatment, and baths are the major applications of EOs in human medical aromatherapy [14].

In veterinary medicine, EOs have the potential to be used as repellents against ectoparasites $[17,18]$. They also have behaviour-modulating properties $[19,20]$. In addition, supplements containing EOs were shown to have a positive effect on atopic dermatitis [21,22], chronic dermatitis [23], pyoderma [24], nasal hyperkeratosis [25], and malodour in dogs [26]. EOs can also be used as ingredients in mouth rinses for their antimicrobial properties $[27,28]$ or in the treatment of abscesses [29].

The antimicrobial activity of EOs can be tested in vitro by agar disc diffusion - related to susceptibility testing of antibiotics or antifungals. This so-called aromatogram is a reproducible and affordable laboratory screening test $[30,31]$.

Studies analysing EO activity against $M$. pachydermatis isolated from canine ears are scarce. Existing in vivo studies analysed the clinical outcome of otitis and the effectivity of single EOs like tea tree oil [32] or the effectivity of a single EO mixture [33] against Malassezia among other microbial agents. A few in vitro studies exist. For example, the activity of 12 EOs against 1 reference strain of $M$. pachydermatis, but also other pathogens, is described [34]. The activity of 11 EOs against 5 clinical isolates and 1 reference strain of $M$. pachydermatis or the activity of cinnamon oil against $20 \mathrm{M}$. pachydermatis isolates from canine otitis was analysed $[35,36]$. Eighteen $M$. pachydermatis isolates from healthy canine ears and 1 reference strain were screened against 14 EOs [37]. Possible synergistic effects between the azole derivative clotrimazole and 5 EOs against $M$. pachydermatis isolated from different localisations were analysed by Bohmova et al. [38]. Khosravi et al. [39] analysed the activity of 6 EOs against Malassezia spp. isolated from different locations of dogs with atopic dermatitis.

The aim of this study was in vitro analyses of the antifungal activity of 22 EOs against 15 clinical $M$. pachydermatis isolates from canine ears deriving from veterinary routine diagnostics to offer scientific background for the veterinary use of EOs in the treatment of Malasseziaassociated otitis externa. To the authors' knowledge, we analysed for the first time a unique panel of 22 EOs by different methods using agar disc diffusion and analyses of volatile activity by vapour assay against a higher number of clinical isolates of $M$. pachydermatis isolated from canine ears. Some of the used EOs overlap with other studies, the other included EOs might bear new potential. Analyses of a larger number of fungal isolates may reveal differences in susceptibility patterns. 


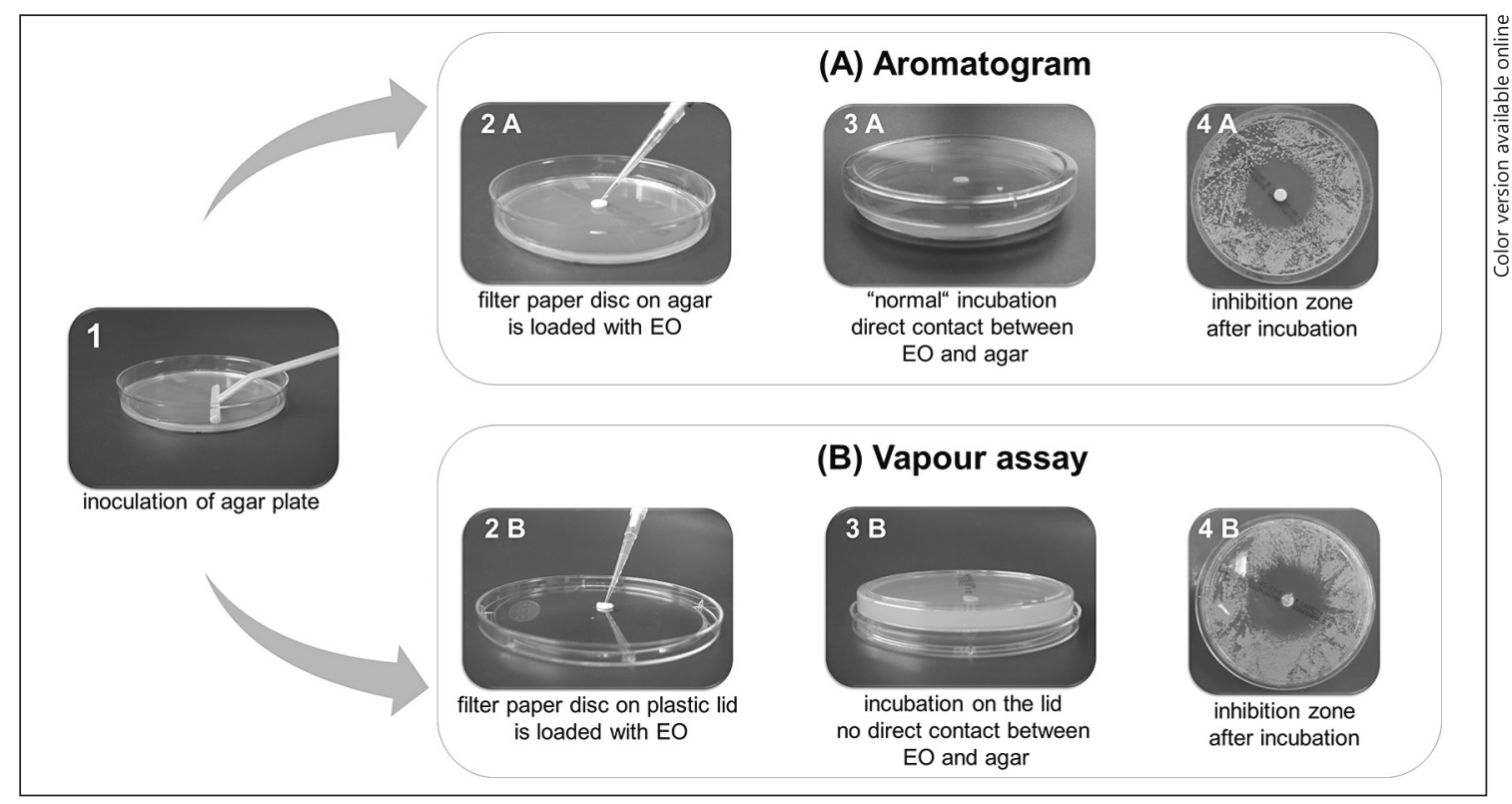

Fig. 1. Experimental setup for aromatogram (agar disc diffusion) (A) in comparison to vapour assay (B). Pictures $4 \mathrm{~A}$ versus $4 \mathrm{~B}$ show the manifestation of inhibition zones after incubation. EO, essential oil.

\section{Materials and Methods}

\section{M. pachydermatis Isolates}

In this study, $15 \mathrm{M}$. pachydermatis strains isolated from ear swabs of dogs were tested for their in vitro susceptibility to EOs. The ear swabs were sent in from veterinarians for routine diagnostics in August 2016. Swabs were streaked out on Sabouraud glucose agar with chloramphenicol and cycloheximide (BD Mycosel Agar; Becton Dickinson GmbH, Heidelberg, Germany). Agar plates were incubated at $36^{\circ} \mathrm{C}$ up to 7 days or until growth of typical $M$. pachydermatis colonies was detected. Afterwards, identification was confirmed by MALDI-TOF mass spectrometry (microflex LT; Bruker Daltonik GmbH, Bremen, Germany). Processing of samples for MALDI-TOF analysis was performed using the ethanolformic acid extraction method according to the manufacturer's standard operating procedure (Bruker Daltonik $\mathrm{GmbH}$ ). Colony material was suspended in $300 \mu \mathrm{L}$ of deionised water (Sigma Aldrich, Taufkirchen, Germany) and mixed by vortexing. Nine hundred microlitres of ethanol (Carl Roth GmbH + Co. KG, Karlsruhe, Germany) was added and vigorously mixed. Afterwards, samples were centrifuged and supernatant was discarded, followed by a similar second centrifugation step. Residual ethanol was carefully removed with a pipette and the cell pellet was dried at room temperature for $3 \mathrm{~min}$. Twenty microlitres of $70 \%$ formic acid (Carl Roth $\mathrm{GmbH}+\mathrm{Co}$. KG) was added to the pellet and vigorously mixed by pipetting. Subsequently, $20 \mu \mathrm{L}$ of acetonitrile (Fluka Chemie GmbH, Buchs, Switzerland) was added and gently mixed. Afterwards, the sample was centrifuged and $1 \mu \mathrm{L}$ of the supernatant was pipetted on a target plate and dried at room temperature. The dried spot was covered with $1 \mu \mathrm{L}$ of IVD HCCA matrix solution (Bruker Daltonik $\mathrm{GmbH}$ ) and dried at room temperature. MALDI-TOF mass spectrometry identification results were automatically classified using the log score values generated by the MALDI Biotyper software procedure (Bruker Daltonik $\mathrm{GmbH})$. All M. pachydermatis isolates included in this study had score values $>2$.

Essential Oil Activity against Malassezia pachydermatis
Malassezia isolates were chosen randomly from identified routine samples under the predetermined condition that they were isolated from canine ears. This random selection resulted in $M$. pachydermatis isolates deriving from dogs of different breeds and mix breeds of both sexes (male: 6; male neutered: 3 ; female: 4 ; female neutered: 2 ), aged from 2 months up to 14 years (mean 7.38 years, standard deviation [SD] 3.46) from different countries (Germany: 11; Switzerland: 1; Austria: 1; Netherlands: 1; Norway: 1).

\section{Aromatogram (Agar Disc Diffusion Test)}

M. pachydermatis colonies were resuspended in sterile $0.9 \%$ $\mathrm{NaCl}$ solution and adjusted to McFarland 0.5. Afterwards, $100 \mu \mathrm{L}$ of $M$. pachydermatis suspension were streaked out on a Sabouraud glucose agar with chloramphenicol and cycloheximide (BD Mycosel Agar; Becton Dickinson GmbH). Subsequently, sterile blank filter paper discs with $6 \mathrm{~mm}$ diameter (BBL ${ }^{\mathrm{TM}}$ Sensi-Disc ${ }^{\mathrm{TM}}$ Susceptibility Test Disc, Blanc Disc; Becton Dickinson $\mathrm{GmbH}$ ) were placed in the middle on the inoculated agar plate and loaded with $10 \mu \mathrm{L}$ of the $\mathrm{EO}$ to be analysed, meaning that $1 \mathrm{EO}$ per agar plate was tested. Plates were kept at room temperature for $30 \mathrm{~min}$ to favour diffusion of EOs over bacterial growth $[40,41]$ and were then incubated for 4 days at $36^{\circ} \mathrm{C}$. After incubation, the radius of the inhibition zones of yeast growth was determined (Fig. 1). Each of the 15 Malassezia isolates was tested in duplicate against all $22 \mathrm{EO}$; for each M. pachydermatis isolate two agar plates per EO were assessed. Thus, two inhibition zone radii per isolate were determined and the mean was calculated and used for classification of EO activity and further descriptive statistical analyses. Growth controls without EO were performed for each Malassezia isolate in duplicate. The in vitro efficacy of EOs was classified as follows: not sensitive for an inhibition radius $<8.0 \mathrm{~mm}$, slightly sensitive for an inhibition radius of $8.0-13.9 \mathrm{~mm}$, moderately sensitive for an inhibition radius of 14.0-19.9 mm, very sensitive for an inhibition radius $\geq 20 \mathrm{~mm}$, and extremely sensitive if total growth on the agar plate was inhibited. The in vitro activity of the 22 commercially available pure EOs listed in Table 1 was analysed against $15 \mathrm{M}$. pachydermatis isolates. EOs can be arranged according to their ef- 


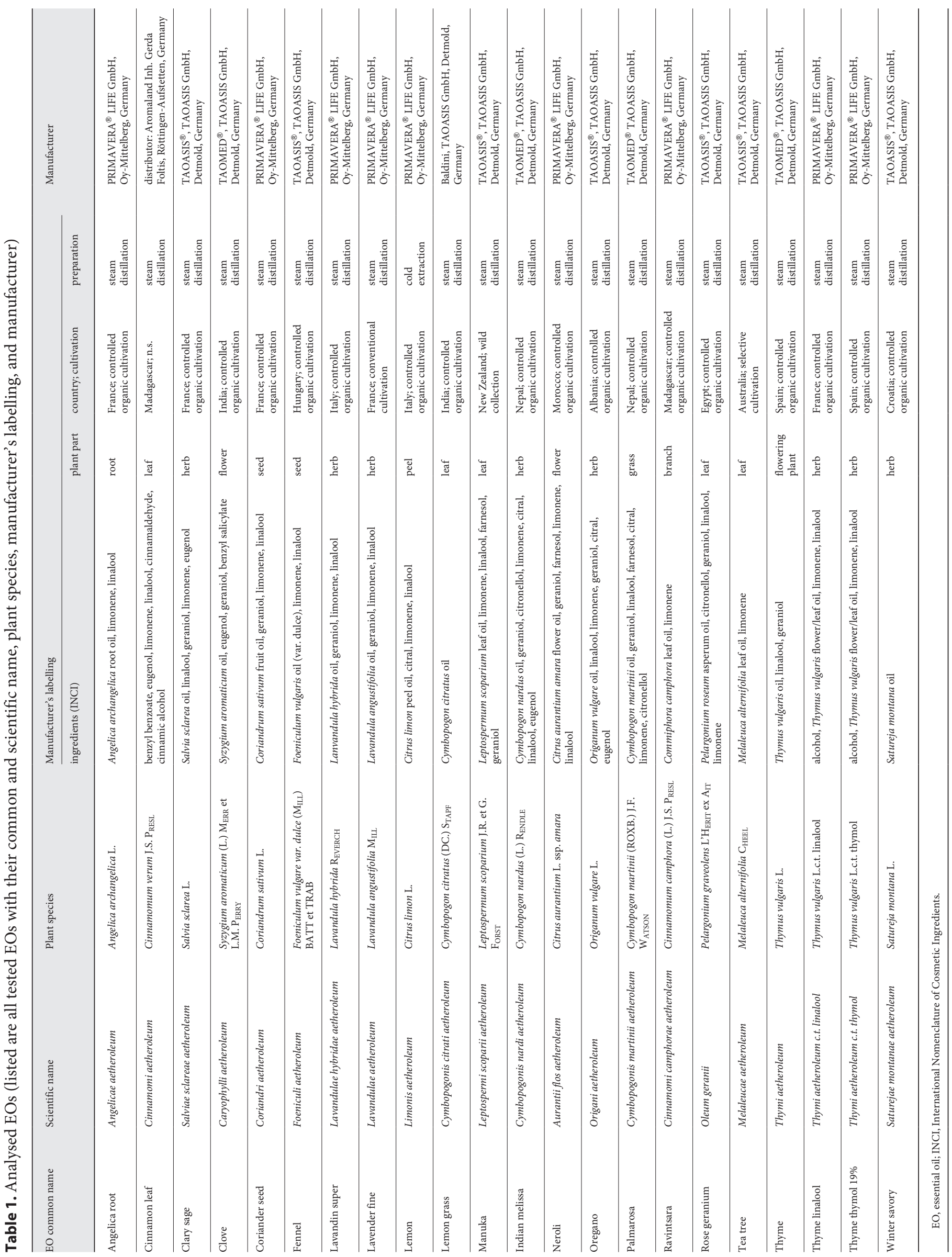


Table 2. Range, median, and IQR of inhibition zone radii of all tested EOs for different experimental setups

\begin{tabular}{|c|c|c|c|c|c|c|c|c|c|c|c|c|}
\hline \multirow[t]{3}{*}{ EO } & \multicolumn{12}{|c|}{ Inhibition zone radius } \\
\hline & \multicolumn{3}{|c|}{ aromatogram $(n=15)$} & \multicolumn{3}{|c|}{ vapour assay $(n=15)$} & \multicolumn{3}{|c|}{ EO $20 \%$ solution $(n=5)$} & \multicolumn{3}{|c|}{ EO $10 \%$ solution $(n=5)$} \\
\hline & $\begin{array}{l}\text { range, } \\
\mathrm{mm}\end{array}$ & $\begin{array}{l}\text { median, } \\
\text { mm }\end{array}$ & IQR & $\begin{array}{l}\text { range, } \\
\mathrm{mm}\end{array}$ & $\begin{array}{l}\text { median, } \\
\text { mm }\end{array}$ & IQR & $\begin{array}{l}\text { range, } \\
\mathrm{mm}\end{array}$ & $\begin{array}{l}\text { median, } \\
\text { mm }\end{array}$ & IQR & $\begin{array}{l}\text { range, } \\
\mathrm{mm}\end{array}$ & $\begin{array}{l}\text { median, } \\
\mathrm{mm}\end{array}$ & IQR \\
\hline Winter savory & $28->40$ & $>40.00$ & 0 & $>40$ & $>40.00$ & 0 & $8.5-13$ & 10.75 & 2.25 & $4-7.5$ & 4.50 & 1.00 \\
\hline Lemon grass & $22.5->40$ & $>40.00$ & 0 & $>40$ & $>40.00$ & 0 & $17-23$ & 19.50 & 2.75 & $6.5-8$ & 8.00 & 0.50 \\
\hline Rose geranium & $22->40$ & $>40.00$ & 0 & $22.5->40$ & $>40.00$ & 0 & $6.5-8$ & 7.00 & 0.50 & 0 & 0 & 0 \\
\hline Oregano & $21->40$ & $>40.00$ & 0 & $>40$ & $>40.00$ & 0 & $8-17$ & 14.50 & 2.50 & $5-7$ & 6.00 & 1.00 \\
\hline Palmarosa & $17.5->40$ & $>40.00$ & 4.50 & $16->40$ & $>40.00$ & 0 & $9.5-17$ & 12.50 & 3.00 & $5-7.5$ & 6.75 & 0.25 \\
\hline Indian melissa & $22.5->40$ & $>40.00$ & 9.75 & $18->40$ & $>40.00$ & 0 & $4.5-8$ & 7.00 & 1.00 & 0 & 0 & 0 \\
\hline Thyme thymol 19\% & $20->40$ & 37.50 & 14.00 & $13->40$ & $>40.00$ & 1.75 & 0 & 0 & 0 & n.t. & & \\
\hline Cinnamon leaf & $25-32$ & 26.00 & 3.50 & $10-35$ & 24.00 & 6.63 & $8-13$ & 9.00 & 1.50 & $4-5.5$ & 4.00 & 0.25 \\
\hline Clove & $25-30$ & 25.00 & 4.88 & $18-32$ & 22.50 & 6.38 & $6-16$ & 8.00 & 1.25 & $0-6$ & 5.00 & 1.00 \\
\hline Thyme & $9.5-26.5$ & 19.75 & 5.50 & $11.5-30$ & 21.50 & 9.75 & 0 & 0 & 0 & n.t. & & \\
\hline Coriander seed & $19-25$ & 19.00 & 3.88 & $12.5-35$ & 17.00 & 6.50 & 0 & 0 & 0 & n.t. & & \\
\hline Thyme linalool & $8-26$ & 16.50 & 5.88 & $4-25.5$ & 13.00 & 5.63 & 0 & 0 & 0 & n.t. & & \\
\hline Manuka & $0-20$ & 15.00 & 2.75 & $4.5-19$ & 10.50 & 5.25 & $4-6.5$ & 5.50 & 0.75 & 0 & 0 & 0 \\
\hline Tea tree & $6-17.5$ & 10.00 & 3.75 & $4-24$ & 7.50 & 4.63 & 0 & 0 & 0 & n.t. & & \\
\hline Lavandin super & $9-22.5$ & 9.00 & 4.38 & $4-17.5$ & 9.50 & 6.25 & 0 & 0 & 0 & n.t. & & \\
\hline Fennel & $8.5-12$ & 8.75 & 1.63 & $0-7.5$ & 0 & 5.50 & 0 & 0 & 0 & n.t. & & \\
\hline Lavender fine & $8.5-15.5$ & 8.00 & 3.13 & $0-17$ & 7.25 & 4.00 & 0 & 0 & 0 & n.t. & & \\
\hline Lemon & $8-11$ & 7.75 & 1.50 & $0-8$ & 4.50 & 6.25 & 0 & 0 & 0 & n.t. & & \\
\hline Clary sage & $7.5-16.5$ & 7.50 & 1.38 & $0-11$ & 5.50 & 6.38 & 0 & 0 & 0 & n.t. & & \\
\hline Angelica root & $7.5-10$ & 7.50 & 2.13 & $0-5$ & 0 & 0 & 0 & 0 & 0 & n.t. & & \\
\hline Ravintsara & $5-9$ & 6.75 & 1.13 & $0-6.5$ & 0 & 5.50 & 0 & 0 & 0 & n.t. & & \\
\hline Neroli & 0 & 0 & 0 & 0 & 0 & 0 & 0 & 0 & 0 & n.t. & & \\
\hline
\end{tabular}

EO, essential oil; IQR, interquartile range; n.t., not tested.

fectivity by the median of all inhibition zone radii means of the tested 15 isolates; in case of equal medians, the higher sum of all inhibition zone radii of each EO counted for higher effectivity (Fig. 1). The chromatographic profiles of EOs specified by manufacturer or product specifications are shown in online supplementary Table 1 (see www.karger.com/doi/10.1159/000504316).

\section{Antifungal Susceptibility Testing}

The activity of antifungal agents was tested simultaneously using agar disc diffusion assay. M. pachydermatis colonies were resuspended in sterile $0.9 \% \mathrm{NaCl}$ solution and adjusted to McFarland 0.5. Afterwards, $100 \mu \mathrm{L}$ of M. pachydermatis suspension were streaked out on a Sabouraud glucose agar with chloramphenicol and cycloheximide. Instead of blank filter paper disc with EOs, an antifungal ring (MASTRING_LAB 504/1; Mast Group Ltd., Merseyside, UK) was placed on the agar. The antifungal ring included among others following agents: amphotericin B $(20 \mu \mathrm{g})$, nystatin $(100 \mu \mathrm{g})$, natamycin $(10 \mu \mathrm{g})$, and 5 -flucytosine $(1 \mu \mathrm{g})$. The diameter of the inhibition zones of yeast growth was determined. Antifungal susceptibility testing of each Malassezia isolate was performed in duplicate, and the mean of duplicate for each isolate was calculated for further descriptive statistical analyses.

\section{Dilution of EOs}

All EOs were diluted in sesame oil as a carrier oil and tested in a $20 \%$ solution for their growth-inhibiting activity in the aromatogram as mentioned above. Growth controls of each Malassezia isolate without $\mathrm{EO}$ were performed in duplicate to exclude an antifungal activity of the carrier oil; additionally, pure sesame oil was used as negative control. The diluted EOs were tested against 5 M. pachydermatis isolates, which were chosen randomly.

Afterwards, all EOs which showed a growth-inhibiting effect in the $20 \%$ solution were tested for their antifungal activity in a $10 \%$ solution. This included cinnamon leaf, clove, lemon grass, manu$\mathrm{ka}$, Indian melissa, oregano, palmarosa, rose geranium, and winter savory oil. The aromatogram of diluted EOs was performed in duplicate; for each tested M. pachydermatis isolate two agar plates per $\mathrm{EO}$ were assessed. Thus, two inhibition zone radii per isolate were determined and the mean was calculated for classification of EO activity and further descriptive statistical analyses.

\section{Vapour Assay}

To analyse the antifungal activity of the volatile components of EOs, the agar disc diffusion test was modified according to Serban et al. [42] and Maruzzella and Sicurella [43]. M. pachydermatis colonies were resuspended in sterile $0.9 \% \mathrm{NaCl}$ solution and adjusted to McFarland 0.5. Afterwards, $100 \mu \mathrm{L}$ of M. pachydermatis suspension were streaked out on a Sabouraud glucose agar with chloramphenicol and cycloheximide. The filter paper disc was placed in the centre of the plastic lid of the agar plate and not on the agar itself and loaded with $10 \mu \mathrm{L}$ of pure EOs. Therefore, the EOs had no direct contact with the agar. Afterwards, plates were incubated standing on their lid and not on their bottom. Thus, only volatile components could exhibit antimicrobial activity. Analysis was performed similar to the agar disc diffusion test by measuring inhibition zone radii (Fig. 1). The vapour assay was performed in duplicate; for each tested $M$. pachydermatis isolate two agar plates per EO were assessed. Thus, two inhibition zone radii per isolate were determined and the mean was calculated for classification 
Fig. 2. Agar disc diffusion: in vitro activity of EOs against $M$. pachydermatis $(n=15)$ using agar disc diffusion. Susceptibility of M. pachydermatis is classified by the size of the zone of inhibition radius in $\mathrm{mm}$ (mean of duplicates): extremely sensitive, no growth at all; very sensitive, $\geq 20 \mathrm{~mm}$; moderately sensitive, $19.9-14.0 \mathrm{~mm}$; slightly sensitive, $13.9-8.0 \mathrm{~mm}$. M. pachydermatis isolates classified as not susceptible (inhibition zone radius $\leq 7.9 \mathrm{~mm}$ ) are not depicted (missing bars). EOs are arranged according to their effectivity in decreasing order from left to right. The median of all inhibition zone radii of the tested 15 isolates was taken for arrangement; in case of equal medians, the higher sum of all inhibition zone radii of each EO counted for higher effectivity. EO, essential oil.

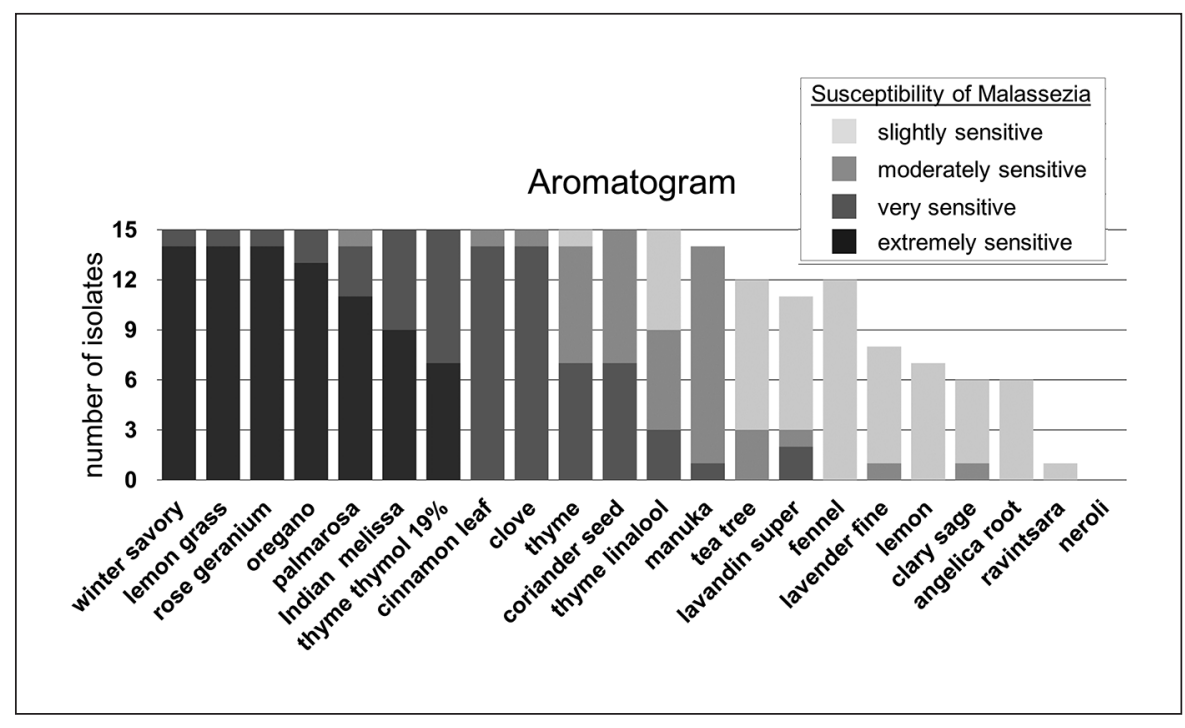

and further descriptive statistical analyses. Growth controls of each Malassezia isolate without EO also incubated on the lid were performed in duplicate.

\section{Statistical Analyses}

Statistical analyses was performed using R [44]. The ShapiroWilk test was used to analyse data for normality $(p<0.05)$. For nonparametric data the mean and interquartile range (IQR) is stated, for parametric data mean and SD.

\section{Results}

\section{Aromatogram}

Agar disc diffusion tests were performed in duplicate and variant inhibition zone radii were detected (the SD of duplicates ranged from 0.0 to $3.5 \mathrm{~mm}$ ). Growth controls showed no inhibition zones. The mean inhibition zone radii of EOs against $M$. pachydermatis are listed in Table 2 (column "Aromatogram"). The tested antifungals, except for 5 -flucytosine, showed inhibition zones. The inhibition zone diameter ranges for amphotericin $\mathrm{B}$ were 10-24 mm (median: 19.0; IQR: 2.0), for nystatin 8$24 \mathrm{~mm}$ (median: 21.0; IQR: 3.5), and for natamycin 8$20 \mathrm{~mm}$ (median: 16.0; IQR: 4.0).

The in vitro efficacy of EOs against $M$. pachydermatis growth showed different degrees and can be arranged by decreasing effectivity (Fig. 2). Winter savory, lemon grass, and rose geranium oil inhibited total growth of $M$. pachydermatis on the agar plate in 14 of 15 cases, oregano oil in 13 of 15 cases. Palmarosa, Indian melissa, thyme thymol $19 \%$, cinnamon leaf, clove, thyme, coriander seed, and thyme linalool oil had an inhibitory effect against all isolates as well by decreasing order.

Manuka, tea tree, lavandin super, fennel, lavender fine, lemon, clary sage, and angelica root oil did not exhibit an- tifungal activity in all cases. Manuka oil was active in 14 of 15 cases, followed by tea tree, lavandin super, and fennel oil. Lavender fine, lemon, clary sage, and angelica root oil had mostly a slight inhibitory effect. Ravintsara oil had only a slight inhibitory effect against 1 isolate of M. pachydermatis. Neroli oil had no inhibitory effect at all.

\section{Dilution of EOs}

Dilution of EOs was performed in duplicate and variant inhibition zone radii were detected (the SD of duplicates ranged from 0.0 to $2.0 \mathrm{~mm}$ ). Growth controls and negative control with carrier sesame oil led to no inhibition zone. The mean inhibition zones of diluted EOs against $M$. pachydermatis are listed in Table 2 (columns "EO 20\% solution" and "EO 10\% solution"). In a 20\% solution some EOs still had a growth-inhibiting effect on $M$. pachydermatis (Fig. 3). Among the diluted EOs, lemon grass was the most potent one. Also effective by decreasing order were oregano, palmarosa, winter savory, cinnamon leaf, clove, Indian melissa, and rose geranium oil. Manuka oil led to very small inhibition zones which were $<8.0 \mathrm{~mm}$ and thus classified as not effective. Thyme thymol 19\%, thyme, coriander seed, thyme linalool, tea tree, lavandin super, fennel, lavender fine, lemon, clary sage, angelica root, ravintsara, and neroli oil showed no inhibition of growth in a $20 \%$ solution.

All EOs which showed an inhibitory effect in the 20\% solution, i.e., cinnamon leaf, clove, lemon grass, manuka, Indian melissa, oregano, palmarosa, and winter savory, were additionally tested in a $10 \%$ solution in sesame oil (Fig. 4). Lemon grass oil was the most effective in the $10 \%$ solution. Cinnamon leaf, clove, oregano, palmarosa, and winter savory oil led to smaller inhibition zones. Manuka, Indian melissa, and rose geranium oil had no growthinhibiting effect in the $10 \%$ solution. 
Fig. 3. Dilution of EOs: in vitro activity of a $20 \%$ EO solution against $M$. pachydermatis $(n=5)$ using agar disc diffusion. Susceptibility of $M$. pachydermatis is classified by the size of the zone of inhibition radius in $\mathrm{mm}$ (mean of duplicates): extremely sensitive, no growth at all; very sensitive, $\geq 20 \mathrm{~mm}$; moderately sensitive, $19.9-14.0$ $\mathrm{mm}$; slightly sensitive, $13.9-8.0 \mathrm{~mm}$. M. pachydermatis isolates classified as not susceptible (inhibition zone radius $\leq 7.9 \mathrm{~mm}$ ) are not depicted (missing bars). EO, essential oil.

Fig. 4. Dilution of EOs: in vitro activity of a $10 \%$ EO solution against $M$. pachydermatis $(n=5)$ using agar disc diffusion. Susceptibility of $M$. pachydermatis is classified by the size of the zone of inhibition radius in $\mathrm{mm}$ (mean of duplicates): extremely sensitive, no growth at all; very sensitive, $\geq 20 \mathrm{~mm}$; moderately sensitive, $19.9-14.0 \mathrm{~mm}$; slightly sensitive, $13.9-8.0 \mathrm{~mm}$. M. pachydermatis isolates classified as not susceptible (inhibition zone radius $\leq 7.9 \mathrm{~mm}$ ) are not depicted (missing bars). EO, essential oil.

Fig. 5. Vapour assay: in vitro activity of EOs against $M$. pachydermatis ( $n=15)$ using vapour assay. Susceptibility of $M$. pachydermatis is classified by the size of the zone of inhibition radius in $\mathrm{mm}$ (mean of duplicates): extremely sensitive, no growth at all; very sensitive, $\geq 20 \mathrm{~mm}$; moderately sensitive, 19.9-14.0 mm; slightly sensitive, 13.9 $8.0 \mathrm{~mm}$. M. pachydermatis isolates classified as not susceptible (inhibition zone radius $\leq 7.9 \mathrm{~mm}$ ) are not depicted (missing bars). EO, essential oil.
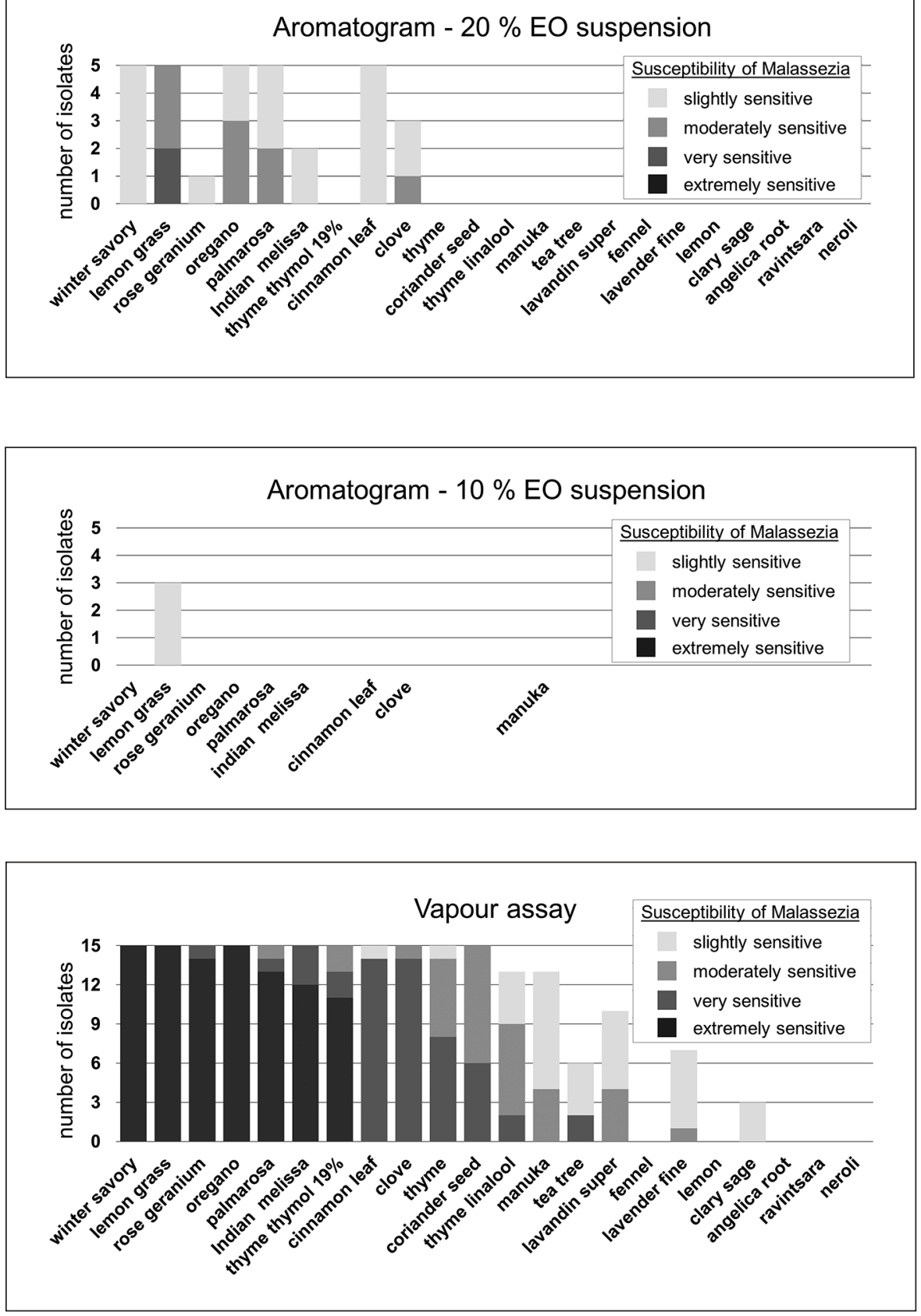

\section{Vapour Assay}

For analysis of volatile components of EOs the vapour assay was performed. Tests were performed in duplicate and variant inhibition zone radii were detected (the SD ranged from 0.0 to $3.5 \mathrm{~mm}$ ). The mean inhibition zones of diluted EOs against $M$. pachydermatis are listed in Table 2 (column "Vapour assay"). The inhibition zones of vapour assay had the same manifestation as observed in the aromatogram (Fig. 1, picture $4 \mathrm{~A}$ vs. $4 \mathrm{~B}$ ), although they may differ in size (Fig. 2 vs. Fig. 5; Table 2, column "Aromatogram" vs. column "Vapour assay").

Eleven of the 22 tested EOs had an antifungal effect against all tested M. pachydermatis isolates in the vapour assay (Fig. 5). Winter savory, lemon grass, and oregano oil inhibited overall growth on agar plates in all cases. Rose geranium, Indian melissa, palmarosa, and thyme thymol 19\% oil also inhibited total growth of isolates in the majority of cases. Cinnamon leaf, clove, thyme, and coriander seed oil showed volatile activity by decreasing order against all $M$. pachydermatis isolates. Thyme linalool, manuka, and lavandin super oil were active against the majority of isolates, while tea tree, lavender fine, and clary sage oil were effective in some cases. Lemon, ravintsara, fennel, angelica root, and neroli oil had no volatile inhibiting effect. 


\section{Discussion and Conclusion}

This study showed an in vitro activity of several EOs against clinical isolates of the yeast M. pachydermatis from canine ears. Especially, winter savory, lemon grass, oregano, palmarosa, and cinnamon leaf oil showed excellent in vitro activity.

EOs exhibit their antifungal activity by different mechanisms at the level of the cell membrane and also within the cell. The structure and function of fungal cell membranes is disrupted and their formation is inhibited. Antifungal components can also lead to dysfunction of fungal mitochondria. In addition, inhibition of biofilm development and anti-quorum sensing of EOs was shown [15].

The antifungal activity of EOs was tested using the agar disc diffusion method (aromatogram). The aromatogram is a measurable method to test the in vitro activity of EOs [30]. The zone of inhibition of fungal growth depends on the sensitivity of Malassezia against the active components of the EOs, but also on the quantity of components which diffuse into the agar. This holds also true for routinely used agar disc diffusion susceptibility testing of antibiotics [45]. The hydrophobic properties of EOs could prevent uniform diffusion through the agar medium [46]. In general, agar disc diffusion provides qualitative results. Since the amount of diffused active components in the agar medium is not known, determination of a minimum inhibitory concentration (MIC) is not possible. Fungistatic or fungicide effects cannot be identified either because inhibition of yeast growth does not necessarily mean fungal death [47].

Up to now, no standardised method for susceptibility testing of EOs exists. Results of studies on the antimicrobial activity of EOs are not easily compared because different methods are used (agar disc diffusion [34, 37], broth dilution [48], microdilution [35, 49-51]). While in agar disc diffusion the activity of an EO depends on diffusion of active components in the agar, in microdilution in ideal circumstances direct contact between all active components of the $\mathrm{EO}$ and the yeast is given. Thus, EOs which are not effective in agar disc diffusion might still have an antimicrobial effect in microdilution.

Even if the method itself is the same, for example agar disc diffusion, factors such as volume of EOs, thickness of the agar layer, solvent, or composition of EOs differ from study to study. The composition of EOs varies due to different plant parts used for extraction, extraction method, natural variation (varieties, subspecies, chemotypes), region of growth, climatic conditions, or time point of harvesting [52-59]. Thus, no constant composition of an EO can be expected. Different composition of an EO can lead to different bioactivity $[57,59,60]$. For the best prediction of EO activity the aromatogram should be performed with exactly the same EO used for therapy, meaning the same charge number. Nevertheless, the aromatogram can be used as a diagnostic tool before therapeutic treatment and can guide clinicians in their choice of an EO [31]. It also can be used as a screening method for potentially active oils $[46,61,62]$. Important is the standardisation of the method used to achieve the best comparability.

The aromatogram (agar disc diffusion) is a reproducible [31], practical, and affordable test for commercial veterinary routine diagnostic laboratories. The used study design is adapted to routine diagnostics and thus might not always keep up with university research procedures. Nevertheless, aromatograms were performed in duplicate, which is comparable to other published studies [3638]. In addition, a higher effort was made to gain better insight into the efficacy of EOs by testing EO dilutions as well as performing the vapour assay, which is not part of daily routine.

Despite the fact that agar disc diffusion might not be the optimal in vitro test, the important conclusion can be drawn that EOs have an inhibiting effect on $M$. pachydermatis isolated from canine ears. Thus, EOs could be a promising candidate for local treatment of $M$. pachydermatis-associated otitis externa.

Although the antifungal activity of EOs is known [15], to our knowledge explicit data on in vitro antifungal activity against clinical isolates of $M$. pachydermatis from canine ears are scarce. As already mentioned, comparison between studies is difficult, and moreover the panel of tested EOs differs from study to study. Subsequently, we only focused on the EOs relevant for comparison with our analyses. One study tested clove, lavender, oregano, winter savory, and tea tree oil for their antifungal activity against $M$. pachydermatis isolated from healthy canine ears $(n=18)$ and 1 type strain at three different concentrations $(0.5,5.0,30 \%)$ [37]. Despite methodological differences in agar disc diffusion regarding the agar media used (Sabouraud dextrose agar with chloramphenicol vs. Sabouraud glucose agar with chloramphenicol and cycloheximide in our study), McFarland standard of inoculum ( 1.0 vs. 0.5 in our study), EO amount ( 15 vs. $10 \mu \mathrm{L}$ in our study), and EO concentration (0.5, 5.0, 30\% EO diluted in liquid paraffin vs. $100,20,10 \%$ EO diluted in sesame oil in our study), the results obtained support each other. Similar to our data, diluted clove and oregano oil showed good activity. Diluted lavender and tea tree oil did not show any activity in both studies. Slightly different results were obtained for winter savory oil, which was active in the $20 \%$ solution in our study, but only showed weak activity in the $30 \%$ solution used by Váczi et al. [37].

Another study showed good in vitro activity of lavandin, lemon, and oregano oil [35]. Different concentrations of those EOs diluted in sweet almond oil (0.6-10\%) were tested by microdilution in semisolid mDixon media 
against 5 clinical isolates of $M$. pachydermatis from canine otitis externa and 1 type strain. Oregano oil with a MIC of $1.3 \%$ in microdilution showed inhibition of growth at a $10 \%$ concentration in the agar disc diffusion used in our study. In contrary, lemon and lavandin oil were active in microdilution (MIC: 1 and 4\%, respectively), but had no effect when used diluted in agar disc diffusion. Clary sage oil in a $10 \%$ solution exhibited no antifungal activity in microdilution in the study by Pistelli [35] nor tested with disc diffusion in our study. Cinnamon EO was effective against $20 \mathrm{M}$. pachydermatis isolates from canine otitis externa tested by microdilution [36]. MIC values of $0.008 \%$ indicate strong activity, which is in accordance with the antifungal activity of the $20 \%$ solution of this EO in our study.

Besides these studies on $M$. pachydermatis isolates from canine ears, data about isolates of unknown localisation, dermatitis, or type strains exist. Clary sage, oregano, lemon grass, tea tree, and thyme oil were tested with agar disc diffusion (in the same procedure as used in our study, except that Müller-Hinton agar was used) against 1 clinical isolate of $M$. pachydermatis from unknown localisation. They showed comparable activity as obtained in our study, whereas the activity of clove and lemon oil was weaker [34]. Lavender and oregano oil tested by microdilution displayed antifungal activity against 5 clinical yeast isolates from dogs with Malassezia-associated dermatitis (MIC: 4.0 and $0.8 \%$, respectively) [49]. Tea tree oil was shown to have in vitro activity against 5 clinical $M$. pachydermatis isolates from canine dermatitis and 4 canine type strains tested by microdilution [50]. A skin cream containing tea tree oil was active tested by microdilution against 1 type strain of $M$. pachydermatis [51]. Tea tree, oregano, and clove oil showed synergistic or additive activity with antifungal clotrimazole against $19 \mathrm{M}$. pachydermatis isolates from different locations (ear, skin interdigital space, perineal area) tested by microdilution [38], emphasising possible benefits of complementary treatment with EOs to conventional medicine.

In addition, some in vivo studies prove that treatment of otitis externa with EOs is definitely a therapeutic option. Application of a 5.0\% tea tree oil lotion into canine ears led to a similar effect as treatment with synthetic antifungal nystatin lotion [32]. An EO mixture of bergamot $(3 \%)$, lavender $(2 \%)$, tea tree (1\%), and roman chamomile (1\%) oil applied twice daily for 2 weeks had a comparable clinical effect to treatment with amoxicillin-clavulanic acid, ciprofloxacin, and ketoconazole. In this study, bacterial and fungal cell count decreased dramatically in the EO-treated group, but only slightly in the control group [33]. Experimentally induced otitis media in rats could be cured in a high proportion of animals by the use of basil oil [63]. Treatment of human otitis patients with ear drops containing clove, lavender, and herb-Robert showed a healing effect comparable to that of ciprofloxacin ear drops (0.3\%) [64].

Also, in cases of Malassezia-associated canine dermatitis, EO-based skin creams showed promising effects in vivo. A skin cream containing $1 \%$ lavender, $0.5 \%$ oregano, and other EOs (0.5-1\%) led to good and long-lasting clinical improvement in dermatitis, whereas conventionally treated dogs had signs of recurrence [49].

Most otitis is polymicrobial and caused by Staphylococcus spp. and M. pachydermatis, but other bacteria can also be involved $[1,3,32]$. In addition, it is discussed whether combined antifungal and antibacterial therapy of otitis externa prevents the switch from yeast to bacterial infection or vice versa if antifungal or antibacterial monotherapy is used [4]. Thus, another positive effect of EOs in treatment could be their additional antibacterial effect. This was already shown in vitro against several bacterial veterinary isolates $[34,36,48,65,66]$. In addition, EOs can exhibit anti-inflammatory potential [67], which might be another positive attribute for treatment of otitis externa.

The volatile antimicrobial properties of EOs, proven in this and other studies [42, 43, 68], could be beneficial for their dispersion in the ear canal. This applies especially for winter savory, lemon grass, rose geranium, oregano, palmarosa, and Indian melissa oil in our study. To our knowledge, this study describes the volatile activity of EOs against $M$. pachydermatis isolated from canine ears for the first time. Others proved the volatile activity of EOs against Malassezia by using different experimental setups. Celery EO was proven to exhibit antimicrobial volatile activity against $M$. furfur by a modified chamber assay [69]. Artemisia annua EO had volatile activity against Malassezia spp.; MICs were lower than those obtained in liquid phase [70].

Several EOs only showed in vitro activity in the agar disc diffusion assay when used undiluted. For therapeutic local application, EOs should not be used undiluted. Due to its popularity, tea tree oil is probably the most investigated EO for compatibility. Instillation of $100 \%$ tea tree oil into the middle ear of guinea pigs led to ototoxicity, whereas a $2 \%$ solution was tolerated well [71]. Also, intratympanic injection of $3 \%$ diluted tea tree oil had no ototoxic side effects in a chinchilla animal model [72]. Treatment of otoacariasis and otitis externa in dogs with a $5 \%$ tea tree oil solution also led to no adverse reaction [32, 73]. A cotton tip wettened in a $50 \%$ tea tree oil solution was even applied as a lid scrub (6 strokes, repeated 3 times total) for treatment of ocular demodex [74]. A 10\% tea tree oil cream for treatment of canine dermatitis was also tolerated well [75]. Additionally, other EOs were shown to lack sensitisation ability and thus safe if used diluted: angelica root, clove oil (Madagascar clarified), coriander, lavender (Yugoslavia), lemon (Sicilian), and lemon grass 
oil were shown to be non-sensitisers in a modified Draize test, including intradermal injection and topical application in guinea pigs [76]. In the same study, citronella oil from Guatemala (0.5\%) with a high content of citronellal (20\%) led to a sensitising effect after two injections, whereas citronella oil from Ceylon (1.0\%) with a low content of citronellal (5\%) did not. Thus, also the composition of the EO used should be taken into consideration before application. Lans et al. [77] documented and validated ethnoveterinary medicine used in British Columbia, Canada. Ear infections in pets were treated with products containing tea tree, thyme, clove, and oregano oil. If used diluted, some EOs showed no adverse side effects applied topically and thus could be a safe alternative for treatment. Nevertheless, the compatibility of the EO intended to be used for therapy should always be clarified before treatment, as should be done with any other drug. There are several records of adverse reactions to EOs [78]. On the other hand, many commercially available ear formulations contain antimicrobial agents which may be ototoxic to the middle and internal ear $[5,79]$.

It is clear that due to different study designs, test methods, and EOs used a comparison of results from different studies is not always possible. The advantage of this study is the broad spectrum of tested EOs against a higher number of clinical isolates of $M$. pachydermatis. Testing more than 1 isolate reveals different susceptibility of $M$. pachydermatis to EOs. This indicates that an EO might help against a $M$. pachydermatis strain isolated from one patient, but might not be the best option for another individual. Thus, no general prediction of effectiveness for one $\mathrm{EO}$ can be made, and it is advisable to perform a mycological examination, including an aromatogram, before treatment.

\section{Statement of Ethics}

The authors have no ethical conflict to disclose. The research was conducted ethically in accordance with the World Medical Association Declaration of Helsinki.

\section{Disclosure Statement}

The authors are employees of Laboklin GmbH \& Co. KG.

\section{Funding Sources}

This study was funded by Laboklin GmbH \& Co. KG.

\section{Author Contributions}

D. Bismarck: conception of the work, design of the work, interpretation of data, drafting of the work, final approval of the version published. A. Dusold: acquisition, analysis and interpretation of data, critical revision of the manuscript for intellectual content, final approval of the version published. A. Heusinger: design of the work, interpretation of data, critical revision of the manuscript for intellectual content, final approval of the version published. E. Müller: conception of the work, critical revision of the manuscript for intellectual content, final approval of the version published.

\section{References}

1 Petrov V, Zhelev G, Marutsov P, Koev K, Gergieva S, Toneva V, et al. Microbiological and antibacterial resistance profile in canine otitis externa - a comparative analysis. Bulg J Vet Med. doi: 10.15547/bjvm.2151.

2 Campbell JJ, Coyner KS, Rankin SC, Lewis TP, Schick AE, Shumaker AK. Evaluation of fungal flora in normal and diseased canine ears. Vet Dermatol. 2010 Dec;21(6):619-25.

3 Oliveira LC, Leite CA, Brilhante RS, Carvalho CB. Comparative study of the microbial profile from bilateral canine otitis externa. Can Vet J. 2008 Aug;49(8):785-8.

4 Bond R. Superficial veterinary mycoses. Clin Dermatol. 2010 Mar;28(2):226-36.

5 Nuttall T. Successful management of otitis externa. In Pract. 2016;38(Suppl 2):17-21.

6 Cafarchia C, Figueredo LA, Iatta R, Montagna MT, Otranto D. In vitro antifungal susceptibility of Malassezia pachydermatis from dogs with and without skin lesions. Vet Microbiol. 2012 Mar;155(2-4):395-8.

7 Nijima M, Kano R, Nagata M, Hasegawa A, Kamata $\mathrm{H}$. An azole-resistant isolate of $\mathrm{Ma}$ lassezia pachydermatis. Vet Microbiol. 2011 Apr;149(1-2):288-90.
8 Jesus FP, Lautert C, Zanette RA, Mahl DL, Azevedo MI, Machado ML, et al. In vitro susceptibility of fluconazole-susceptible and -resistant isolates of Malassezia pachydermatis against azoles. Vet Microbiol. 2011 Aug; 152(1-2):161-4.

9 Fera MT, La Camera E, De Sarro A. New triazoles and echinocandins: mode of action, in vitro activity and mechanisms of resistance. Expert Rev Anti Infect Ther. 2009 Oct;7(8): 981-98.

10 Shin B, Park W. Zoonotic Diseases and Phytochemical Medicines for Microbial Infections in Veterinary Science: Current State and Future Perspective. Front Vet Sci. 2018 Jul; 5(5): 166

11 Russo R, Autore G, Severino L. Pharmacotoxicological aspects of herbal drugs used in domestic animals. Nat Prod Commun. 2009 Dec;4(12):1777-84.

12 Hahn I, Zitterl-Eglseer K, Franz C. Phytomedicine in dogs and cats: web-based survey among veterinarians in Austria, Germany and Switzerland. Schweiz Arch Tierheilkd. 2005 Mar;147(3):135-41. German.
13 Ali B, Al-Wabel NA, Shams S, Ahamad A, Khan SA, Anwar F. Essential oils used in aromatherapy: A systemic review. Asian Pac J Trop Biomed. 2015;8(5):601-11.

14 Sharifi-Rad J, Sureda A, Tenore GC, Daglia M, Sharifi-Rad M, Valussi M, et al. Biological Activities of Essential Oils: From Plant Chemoecology to Traditional Healing Systems. Molecules. 2017 Jan;22(1):E70.

15 Nazzaro F, Fratianni F, Coppola R, Feo V. Essential Oils and Antifungal Activity. Pharmaceuticals (Basel). 2017 Nov;10(4):E86.

16 Reichling J, Schnitzler P, Suschke U, Saller R. Essential oils of aromatic plants with antibacterial, antifungal, antiviral, and cytotoxic properties - an overview. Forsch Komplement Med. 2009;2(16):79-90.

17 George DR, Finn RD, Graham KM, Sparagano OA. Present and future potential of plantderived products to control arthropods of veterinary and medical significance. Parasit Vectors. 2014 Jan;7(7):28.

18 Lee MY. Essential oils as repellents against arthropods. Biomed Res Int. 2018 Oct;2018: 6860271 . 
19 Ferguson CE, Kleinman HF, Browning J. Effect of Lavender Aromatherapy on AcuteStressed Horses. J Equine Vet Sci. 2013;1(33): 67-9.

20 Heitman K, Rabquer B, Heitman E, Streu C, Anderson P. The Use of Lavender Aromatherapy to Relieve Stress in Trailered Horses. J Equine Vet Sci. 2018;63:8-12.

21 Tretter S, Mueller RS. The influence of topical unsaturated fatty acids and essential oils on normal and atopic dogs. J Am Anim Hosp Assoc. 2011 Jul-Aug;47(4):236-40.

22 Blaskovic M, Rosenkrantz W, Neuber A, Sauter-Louis C, Mueller RS. The effect of a spoton formulation containing polyunsaturated fatty acids and essential oils on dogs with atopic dermatitis. Vet J. 2014 Jan;199(1):3943.

23 Fitzi J, Fürst-Jucker J, Wegener T, Saller R, Reichling J. Phytotherapy of chronic dermatitis and pruritus of dogs with a topical preparation containing tea tree oil (Bogaskin). Schweiz Arch Tierheilkd. 2002 May;144(5): 223-31.

24 Bensignor E, Fabriès L, Bailleux L. A splitbody, randomized, blinded study to evaluate the efficacy of a topical spray composed of essential oils and essential fatty acids from plant extracts with antimicrobial properties. Vet Dermatol. 2016 Dec;27(6):464-e123.

25 Catarino M, Combarros-Garcia D, Mimouni P, Pressanti C, Cadiergues MC. Control of canine idiopathic nasal hyperkeratosis with a natural skin restorative balm: a randomized double-blind placebo-controlled study. Vet Dermatol. 2018 Apr;29(2):134-e53.

26 Meason-Smith C, Older CE, Ocana R, Dominguez B, Lawhon SD, Wu J, et al. Novel association of Psychrobacter and Pseudomonas with malodour in bloodhound dogs, and the effects of a topical product composed of essential oils and plant-derived essential fatty acids in a randomized, blinded, placebo-controlled study. Vet Dermatol. 2018 Dec;29(6): 465-e158.

27 Girão VC, Nunes-Pinheiro DC, Morais SM, Sequeira JL, Gioso MA. A clinical trial of the effect of a mouth-rinse prepared with Lippia sidoides Cham essential oil in dogs with mild gingival disease. Prev Vet Med. 2003 May; 59(1-2):95-102.

28 Low SB, Peak RM, Smithson CW, Perrone J, Gaddis B, Kontogiorgos E. Evaluation of a topical gel containing a novel combination of essential oils and antioxidants for reducing oral malodor in dogs. Am J Vet Res. 2014 Jul; 75(7):653-7.

29 Kawakami E, Washizu M, Hirano T, Sakuma M, Takano M, Hori T, et al. Treatment of prostatic abscesses by aspiration of the purulent matter and injection of tea tree oil into the cavities in dogs. J Vet Med Sci. 2006 Nov; 68(11):1215-7

30 Werner M, von Braunschweig R. Praxis Aromatherapie: Grundlagen, Steckbriefe, Indikationen. Stuttgart: Haug; 2006.

31 Zimmermann E. Aromatherapie für Pflegeund Heilberufe: Kursbuch für Ausbildung und Praxis. 6th ed. Stuttgart: Haug; 2018.

32 Neves RC, Makino H, Cruz TP, Silveira MM, Sousa VR, Dutra V, et al. In vitro and in vivo efficacy of tea tree essential oil for bacterial and yeast ear infections in dogs. Pesqui Vet Bras. 2018;8(38):1597-607.

33 Kim SH, Kim S, Kyou HK, Kim DH. Efficacy of aromatherapy for the treatment of otitis externa in dogs. Korean J Vet Res. 2009;1(49): 85-9.

34 Rusenova NP. Antimicrobial activities of twelve essential oils against microorganisms of veterinary importance. Trakia J Sci. 2009; 1(7):37-43.

35 Pistelli L. Antimycotic activity of some aromatic plants essential oils against canine isolates of Malassezia pachydermatis: an in vitro assay. Open Mycol J. 2012;1(6):17-21.

36 Sim JX, Khazandi M, Pi H, Venter H, Trott DJ, Deo P. Antimicrobial effects of cinnamon essential oil and cinnamaldehyde combined with EDTA against canine otitis externa pathogens. J Appl Microbiol. 2019 Jul;127(1): 99-108.

37 Váczi P, Čonková E, Marcinčáková D, Sihelská Z. Antifungal Effect of Selected Essential Oils on Malassezia pachydermatis Growth. Folia Vet. 2018;2(62):67-72.

38 Bohmova E, Conkova E, Harcarova M, Sihels$\mathrm{ka} \mathrm{Z}$. Interactions between Clotrimazole and selected essential oils against Malassezia pachydermatis clinical isolates. Pol J Vet Sci. 2019 Mar;22(1):173-5.

39 Khosravi AR, Shokri H, Fahimirad S. Efficacy of medicinal essential oils against pathogenic Malassezia sp. isolates. J Mycol Med. 2016 Mar;26(1):28-34.

40 Janssen AM, Chin NL, Scheffer JJ, Baerheim Svendsen A. Screening for antimicrobial activity of some essential oils by the agar overlay technique. Pharm Weekbl Sci. 1986 Dec;8(6): 289-92.

41 Onawunmi GO, Yisak WA, Ogunlana EO. Antibacterial constituents in the essential oil of Cymbopogon citratus (DC.) Stapf. J Ethnopharmacol. 1984 Dec;12(3):279-86.

42 Serban ES, Ionescu M, Matinca D, Maier CS, Bojita MT. Screening of the antibacterial and antifungal activity of eight volatile essential oils. Farmacia. 2011;3(59):440-6.

43 Maruzzella JC, Sicurella NA. Antibacterial Activity of Essential Oil Vapors. J Am Pharm Assoc (Sci Ed). 1960;11(49):692-4.

44 R Development Core Team. R: A language and environment for statistical computing: reference index. Vienna: R Foundation for Statistical Computing; 2010.

45 Jorgensen JH, Ferraro MJ. Antimicrobial susceptibility testing: a review of general principles and contemporary practices. Clin Infect Dis. 2009 Dec;49(11):1749-55.

46 Janssen AM, Scheffer JJ, Baerheim Svendsen A. Antimicrobial activity of essential oils: a 1976-1986 literature review. Aspects of the test methods. Planta Med. 1987 Oct;53(5): 395-8.

47 Balouiri M, Sadiki M, Ibnsouda SK. Methods for in vitro evaluating antimicrobial activity: A review. J Pharm Anal. 2016 Apr;6(2):71-9.

48 Niculae M, Köbölkuti LB, Czirjak GA, Cadar D, Bolfa P, Ungvari A. Evaluation of some herbal extractions' antimicrobial potential against Staphylococcus spp. strains associated with canine otitis externa. Bull Univ Agric Sci Vet Med Cluj-Napoca Vet Med. 2007;64(1/2): 597.
49 Nardoni S, Mugnaini L, Pistelli L, Leonardi M, Sanna V, Perrucci S, et al. Clinical and mycological evaluation of an herbal antifungal formulation in canine Malassezia dermatitis. J Mycol Med. 2014 Sep;24(3):234-40.

50 Weseler A, Geiss HK, Saller R, Reichling J. Antifungal effect of Australian tea tree oil on Malassezia pachydermatis isolated from canines suffering from cutaneous skin disease. Schweiz Arch Tierheilkd. 2002 May;144(5): 215-21.

51 Han JI, Park SJ, Kim SG, Park HM. Antimicrobial effects of topical skin cream containing natural oil mixtures against Staphylococcus pseudintermedius and Malassezia pachydermatis. Veterinarni Medicina. 2016;60(4): 202-7.

52 Barbosa LC, Demuner AJ, Clemente AD, Paula VF, Ismail FM. Seasonal variation in the composition of volatile oils from Schinus terebinthifolius raddi. Quim Nova. 2007;8(30): 1959-65.

53 Bernotienè G. Essential oil composition variability in sage(Salvia officinalis L.). Chemija. 2007;4(18):38-43.

54 Demuner AJ, Barbosa LC, Magalhaes CG, da Silva CJ, Maltha CR, Pinheiro AL. Seasonal variation in the chemical composition and antimicrobial activity of volatile oils of three species of Leptospermum (Myrtaceae) grown in Brazil. Molecules. 2011 Jan;16(2): 1181-91.

55 Marotti M, Piccaglia R, Giovanelli E, Deans SG, Eaglesham E. Effects of planting time and mineral fertilization on peppermint (mentha $\mathrm{x}$ piperita 1.) essential oil composition and its biological activity. Flavour Fragr J. 1994;9(3): 125-9.

56 McGimpsey JA, Douglas MH, van Klink JW, Beauregard DA, Perry NB. Seasonal variation in essential oil yield and composition from naturalized Thymus vulgaris L. in New Zealand. Flavour Fragr J. 1994;9(6):347-52.

57 Lis-Balchin M, Deans SG, Eaglesham E. Relationship between bioactivity and chemical composition of commercial essential oils. Flavour Fragr J. 1998;13(2):98-104.

58 Russo A, Formisano C, Rigano D, Senatore F, Delfine S, Cardile V, et al. Chemical composition and anticancer activity of essential oils of Mediterranean sage (Salvia officinalis L.) grown in different environmental conditions. Food Chem Toxicol. 2013 May;55(55):42-7.

59 Nostro A, Germanò MP, D’Angelo V, Marino A, Cannatelli MA. Extraction methods and bioautography for evaluation of medicinal plant antimicrobial activity. Lett Appl Microbiol. 2000 May;30(5):379-84.

60 Zhang L, Yang Z, Wei J, Su P, Pan W, Zheng $\mathrm{X}$, et al. Essential oil composition and bioactivity variation in wild-growing populations of Curcuma phaeocaulis Valeton collected from China. Ind Crops Prod. 2017;103:27482.

61 Burt S. Essential oils: their antibacterial properties and potential applications in foods - a review. Int J Food Microbiol. 2004 Aug;94(3): 223-53.

62 Chao S, Young G, Oberg C, Nakaoka K. Inhibition of methicillin-resistant Staphylococcus aureus (MRSA) by essential oils. Flavour Fragr J. 2008;23(6):444-9. 
63 Kristinsson KG, Magnusdottir AB, Petersen $\mathrm{H}$, Hermansson A. Effective treatment of experimental acute otitis media by application of volatile fluids into the ear canal. J Infect Dis. 2005 Jun;191(11):1876-80.

64 Panahi Y, Akhavan A, Sahebkar A, Hosseini SM, Taghizadeh M, Akbari H, et al. Investigation of the effectiveness of Syzygium aromaticum, Lavandula angustifolia and Geranium robertianum essential oils in the treatment of acute external otitis: a comparative trial with ciprofloxacin. J Microbiol Immunol Infect. 2014 Jun;47(3):211-6.

65 Bismarck D, Schneider M, Müller E. Antibakterielle In-vitro-Wirksamkeit ätherischer Öle gegen veterinärmedizinisch relevante Keime klinischer Isolate von Hunden, Katzen und Pferden. Complement Med Res. 2017;24(3) 153-63.

66 Ebani VV, Nardoni S, Bertelloni F, Najar B, Pistelli L, Mancianti F. Antibacterial and Antifungal Activity of Essential Oils against Pathogens Responsible for Otitis Externa in Dogs and Cats. Medicines (Basel). 2017 Apr; 4(2):E21.

67 de Lavor ÉM, Fernandes AW, de Andrade Teles RB, Leal AE, de Oliveira Júnior RG, Gama E Silva M, et al. Essential Oils and Their Major Compounds in the Treatment of Chronic Inflammation: A Review of Antioxidant Potential in Preclinical Studies and Molecular Mechanisms. Oxid Med Cell Longev. 2018 Dec;2018:6468593.
68 Inouye S, Uchida K, Maruyama N, Yamaguchi $\mathrm{H}$, Abe S. A novel method to estimate the contribution of the vapor activity of essential oils in agar diffusion assay. Nippon Ishinkin Gakkai Zasshi. 2006;47(2):91-8.

69 Chee HY, Lee MH. In vitro Activity of Celery Essential Oil against Malassezia furfur. Mycobiology. 2009 Mar;37(1):67-8.

70 Santomauro F, Donato R, Pini G, Sacco C, Ascrizzi R, Bilia AR. Liquid and Vapor-Phase Activity of Artemisia annua Essential Oil against Pathogenic Malassezia spp. Planta Med. 2018 Feb;84(3):160-7.

71 Zhang SY, Robertson D. A study of tea tree oil ototoxicity. Audiol Neurotol. 2000 Mar-Apr; 5(2):64-8.

72 Bezdjian A, Mujica-Mota MA, Azzi M, Daniel SJ. Assessment of ototoxicity of tea tree oil in a chinchilla animal model. Int J Pediatr Otorhinolaryngol. 2014 Dec;78(12):2136-9.

73 Neves RCSM, Ferraz RHS, Mendonça AJ, Lima SR, Cruz FACS, Rosa JG, et al. Acaricide effect of the Melaleuca alternifolia essential oil on Otodectes cynotis. Rev Bras Ci Vet. 2012; 19(3):144-8. Portuguese.
74 Gao YY, Di Pascuale MA, Li W, BaradaranRafii A, Elizondo A, Kuo CL, et al. In vitro and in vivo killing of ocular Demodex by tea tree oil. Br J Ophthalmol. 2005 Nov;89(11):146873.

75 Reichling J, Fitzi J, Hellmann K, Wegener T, Bucher S, Saller R. Topical tea tree oil effective in canine localised pruritic dermatitis - a multi-centre randomised double-blind controlled clinical trial in the veterinary practice. Dtsch Tierarztl Wochenschr. 2004 Oct; 111(10):408-14.

76 Sharp DW. The sensitization potential of some perfume ingredients tested using a modified Draize procedure. Toxicology. 1978 Mar;9(3):261-71.

77 Lans C, Turner N, Khan T. Medicinal plant treatments for fleas and ear problems of cats and dogs in British Columbia, Canada. Parasitol Res. 2008 Sep;103(4):889-98.

78 Genovese AG, McLean MK, Khan SA. Adverse reactions from essential oil-containing natural flea products exempted from Environmental Protection Agency regulations in dogs and cats. J Vet Emerg Crit Care (San Antonio). 2012 Aug;22(4):470-5.

79 Merchant SR. Ototoxicity. Vet Clin North Am Small Anim Pract. 1994 Sep;24(5):97180. 